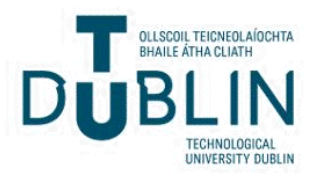

Technological University Dublin ARROW@TU Dublin

Articles

2019-6

\section{Clinical Accuracy of the Nidek ARK-1 Autorefractor}

\author{
Nabin Paudel \\ Technological University Dublin, nabin.paudel@TUDublin.ie \\ Sameep Adhikari \\ Drishti Eye Care Centre, Kalanki, Kathmandu, Nepal \\ Ajit Thakur \\ Drishti Eye Care Centre, Kalanki, Kathmandu, Nepal
}

See next page for additional authors

Follow this and additional works at: https://arrow.tudublin.ie/otpomart

Part of the Optometry Commons

\section{Recommended Citation}

Paudel, N. et al (2019) Clinical Accuracy of the Nidek ARK-1 Autorefractor, Optometry and Vision Science: June 2019 - Volume 96 - Issue 6 - p 407-413 doi: 10.1097/OPX.0000000000001386

This Article is brought to you for free and open access by ARROW@TU Dublin. It has been accepted for inclusion in Articles by an authorized administrator of ARROW@TU Dublin. For more information, please contact arrow.admin@tudublin.ie, aisling.coyne@tudublin.ie, gerard.connolly@tudublin.ie.

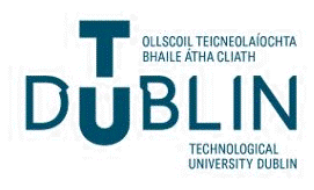




\section{Authors}

Nabin Paudel, Sameep Adhikari, Ajit Thakur, Bhairaja Shrestha, and James Loughman

This article is available at ARROW@TU Dublin: https://arrow.tudublin.ie/otpomart/80 


\title{
Clinical Accuracy of the Nidek ARK-1 Autorefractor
}

\author{
Nabin Paudel, PhD, ${ }^{1,2 *}$ Sameep Adhikari, BOptom, ${ }^{1}$ Ajit Thakur, MOptom, ${ }^{1}$ Bhairaja Shrestha, MD, ${ }^{1}$ and James Loughman, $\mathrm{PhD}^{2}$
}

\begin{abstract}
SIGNIFICANCE: Autorefractors are commonly used by eye care practitioners worldwide as a starting point for clinical prescribing and by researchers as an instrument to study development of refractive errors and accommodation. This study demonstrates that the Nidek ARK-1 provides a reasonable and repeatable estimate of refractive error.

PURPOSE: The purposes of this study were (a) to compare refraction measurements of the Nidek ARK-1 (2016 release) autorefractor with that of subjective refraction and retinoscopy performed by an experienced clinician and (b) to determine the intratest and intertest variability of autorefraction measures taken using the ARK-1 autorefractor.

METHODS: Sixty-seven adult patients aged 18 to 69 years underwent retinoscopy, subjective refraction, and ARK-1 autorefraction on a same day by a single clinician. A separate subset of 14 participants was invited for the repeatability and reproducibility study. Both eyes of each participant were included in the analysis.

RESULTS: A statistically significant (but not clinically significant) positive spherical difference was observed between the ARK-1 and subjective refraction $(P=.003)$. Spherical equivalent refractive errors were statistically similar between the ARK-1 and subjective refraction $(P=.20)$. A statistically and clinically significant difference was observed in the cylindrical component between the ARK and subjective refraction $(P<.01)$. No statistically significant difference was observed between the ARK and subjective refraction in both the horizontal $\left(J_{0} ; P=.08\right)$ and oblique cylindrical vector $\left(J_{45} ; P=.96\right)$. Bland-Altman analysis revealed that the $95 \%$ limits of agreement were widest between the ARK and subjective refraction in all of the refractive components $(-0.60$ to 0.89 diopter for spherical component, -0.80 to 0.69 diopter for spherical equivalent, and -0.98 to 0.30 diopter for cylindrical component). The intertest and intratest variability of the ARK-1 was small.
\end{abstract}

CONCLUSIONS: The Nidek ARK-1 autorefractor is a useful clinical tool that provides a reasonable and repeatable estimation of refractive error in adults.

Optom Vis Sci 2019;00:00-00. doi:10.1097/OPX.0000000000001386

Copyright $\odot 2019$ American Academy of Optometry

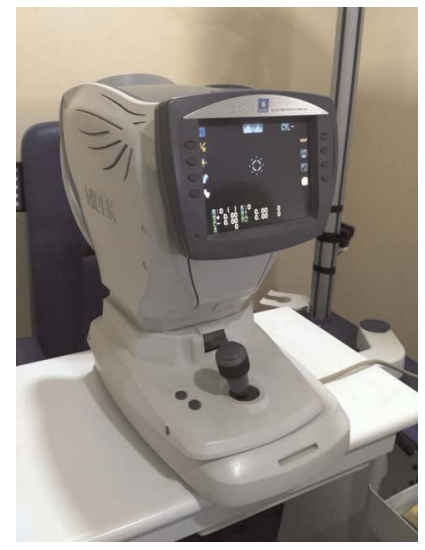
Author Affiliations:
${ }^{1}$ Drishti Eye Care Centre, Kalanki, Kathmandu, Nepal
${ }^{2}$ Centre for Eye Research Ireland, Technological University Dublin,
Dublin, Ireland
*nabin.paudel@dit.ie

Autorefraction has been an integral procedure in optometric practice for many years. It is commonly used as a starting point for subjective refraction in clinical practice ${ }^{1,2}$ and to study accommodation status ${ }^{3}$ and the development of refractive errors in research. ${ }^{4}$ Most of the commercially available autorefractors are designed as a closed-view apparatus whereby a fixation target is located within the system and a combination of lenses is incorporated to relax accommodation. This arrangement does not represent a natural viewing environment and can therefore lead to the stimulation of accommodation due to proximity of the surrounding or awareness of being in an enclosed surrounding known as proximal accommodation. ${ }^{5,6}$ This accommodation interferes with the true measurement of refractive error of the eyes and hence leads to inaccuracies in the results. A variety of autorefractors are available, each based on one of a number of different design principles. New models of autorefractor are released regularly, generally incorporating upgraded design or operational features to overcome measurement challenges such as minimizing the influence of accommodation and eye movements. From a clinical and research perspective, devices that provide rapid measurements, incorporate features to optimize measurement accuracy and reliability, provide natural unobstructed binocular viewing, and are less influenced by factors such as accommodation and eye movements are desirable. ${ }^{7}$

Many previous studies have compared the performance of autorefractors with that of subjective refraction and retinoscopy. ${ }^{8-12}$
Although some level of disagreement is to be expected between different methods, the levels of agreement are variable. Because different autorefractors work on different design principles, it is necessary to compare the performance of each newly released instrument with other models ${ }^{13}$ and also with traditional refraction methods such as retinoscopy and subjective refraction. The level of agreement between different methods and the accuracy and the validity of the instrument will determine the confidence of the clinician or the researcher to trust the results obtained with the instrument, whether be it for clinical prescribing or for research purposes.

The Nidek ARK-1 (Nidek Technologies, Gamagori, Japan) is an autoref/keratometer platform based on the Scheiner disc principle. ${ }^{14}$ It combines pupillography, keratometry, and autorefraction. The ARK-1 is a relatively new addition to the autorefractor market (released in 2016). It uses a large pupil zone imaging method and has measurement ranges of -30 to +25 diopter sphere, up to 12 diopter cylinder, and 0 to $180^{\circ}$ axis. The autorefractor incorporates a super luminescent diode that provides a clear and sharp image compared with older designs and a highly sensitive charge-coupled device that is stated to allow the autorefractor to perform measurement in densely cataractous eyes. ${ }^{15}$

The purpose of this study was twofold. First, the study was designed to compare the autorefraction performance of the Nidek ARK-1 autorefractor with the routinely used standard refraction protocolretinoscopy and subjective refraction—-performed by an experienced 
eye care professional in noncyclopleged adult eyes. Second, the intratest and intertest variability of autorefraction measures applied to the autorefraction function of the ARK-1 was determined.

\section{METHODS}

This was a cross-sectional study whereby 67 patients attending the Drishti Eye Care Center, a tertiary-level eye care center in Kathmandu, Nepal, were enrolled. The research was approved by the ethical committee of the Nepal Health Research Council, and the study was conducted in accordance with the tenets of the Declaration of Helsinki. Verbal and written informed consents were obtained from each participant before being enrolled into the study. Subjects were included only if they were free of ocular pathology. All of the refraction procedures were performed under noncycloplegic conditions. Retinoscopy was performed first, followed by subjective refraction. Either the retinoscopy finding or the patient's previous prescription (if available) was used as the starting point for subjective refraction. Subjective refraction was conducted with a trial frame with vertex distance kept to a minimum. Cylindrical power and axis were refined using a 0.25-diopter Jackson cross cylinder. Refraction of each eye was performed monocularly followed by binocular balancing using the alternate occlusion method. The highest plus sphere and the lowest minus cylindrical power that provided the best acuity and comfortable vision were considered as the end point. Subjective refraction was recorded to the closest 0.25 diopter sphere, 0.25 diopter cylinder, and $2.5^{\circ}$. To minimize the risk of bias, autorefraction was conducted by the same examiner (NP) but after subjective refraction was completed. The autorefractor was calibrated daily according to the manufacturer's guidelines using a schematic model eye (-5.00 diopter sphere). Participants were instructed to look at the internal fixation target (balloon). Three measurements of each eye were taken over an approximate 6 second time frame, and the readings were manually averaged by the clinician. Intratest and intertest variability of the ARK-1 was determined in a separate group of 14 individuals using two methods, determining the standard deviation of seven consecutive readings (intratest) and retesting the same eye within a duration of 2 weeks (intertest).

\section{Data Analysis}

Assuming an effect size of 0.4 , our sample size calculations $\left(G *\right.$ Power ${ }^{16}$ ) indicate that 67 eyes are sufficient for a power of $90 \%$ with a two-sided paired $t$ test, adopting a significance value of $5 \%$.

Both eyes of each participant were included in the data analysis. There was no significant variation in the measured refractive error between eyes (intraclass correlation between two eyes close to 1 for all measures). Statistical analyses therefore were computed using averaged data from both eyes as suggested by Armstrong. ${ }^{17}$

Refraction measurements were compared in the form of mean sphere, cylinder, and spherical equivalent. Spherical equivalent was calculated by adding half of the cylindrical power to the spherical component. Measurements were also subjected to vector analysis for comparison. Vector analysis is an established method for evaluating refractive error data, as it considers magnitude and direction of the cylinder of astigmatism for statistical calculation of refractive errors. ${ }^{18}$ Vector analysis was conducted using the formulas suggested by Thibos et al. ${ }^{18}$ represented as follows, whereby $J_{0}$ represents the horizontal cylindrical vector and $J_{45}$ represents the oblique cylindrical vector.

$$
\begin{aligned}
& J_{0}=-(\text { cylinder } / 2) \cos (2 \times \text { axis }) \\
& J_{45}=-(\text { cylinder } / 2) \sin (2 \times \text { axis })
\end{aligned}
$$

Bland-Altman plots ${ }^{19}$ of the sphere, cylinder, spherical equivalent, and $J_{0}$ and $J_{45}$ were used to demonstrate the limits of agreement between retinoscopy, subjective refraction, and autorefraction values, whereas mean differences were assessed using a paired-samples $t$ test. SPSS v.21 statistical software was used for data analysis (SPSS, Chicago, IL). MedCalc statistical software was used to plot the Bland-Altman charts. ${ }^{20}$

\section{RESULTS}

One hundred thirty-four eyes of 67 participants (54\% male) were assessed. Mean \pm SD age of the participants was $32 \pm 13$ years (range, 18 to 69 years). Subjectively assessed mean spherical equivalent refractive errors ranged from +3.00 to -6.25 diopter sphere. The maximal astigmatism was 3.00 diopter cylinder. Of the eyes with astigmatism ( $n=87$ ), $56 \%$ were against the rule, $35 \%$ were with the rule, and the rest were oblique. Twenty-eight eyes of 14 participants with a mean age of $26 \pm 6$ years were subjected to the repeatability and reproducibility analysis. Subjectively assessed spherical equivalent refractive error for this analysis ranged between +0.50 and -1.50 diopter sphere. The maximal astigmatism was -1.50 diopter cylinder.

\section{Spherical Component Analysis}

The Nidek ARK-1 statistically significantly underestimated the spherical component as compared with subjective refraction and retinoscopy (Table 1; Figs. 1 and 2). The 95\% limits of agreement in the spherical component between the ARK and retinoscopy, the

TABLE 1. Mean difference and significance in various refractive components between ARK-1, retinoscopy, and subjective refraction

\begin{tabular}{lcccccc}
\hline & \multicolumn{5}{c}{ Mean \pm SD difference (95\% Cl) and significance } \\
\cline { 2 - 6 } Refractive components & ARK vs. RET & $\boldsymbol{P}$ & ARK vs. SUB & $\boldsymbol{P}$ & RET vs. SUB \\
\hline Spherical component & $0.09 \pm 0.35(0.00$ to 0.18$)$ & $.03^{*}$ & $0.14 \pm 0.38(0.05$ to 0.23$)$ & $.003^{*}$ & $0.04 \pm 0.25(-0.01$ to 0.25$)$ & .16 \\
Cylindrical component & $-0.15 \pm 0.26(-0.21$ to -0.08$)$ & $<.001^{*}$ & $-0.33 \pm 0.32(-0.41$ to -0.25$)$ & $<.001^{*}$ & $-0.18 \pm 0.31(-0.26$ to -0.10$)<.001^{*}$ \\
Spherical equivalent (M) & $0.00 \pm 0.33(-0.07$ to 0.08$)$ & .95 & $-0.05 \pm 0.38(-0.15$ to 0.03$)$ & .20 & $-0.06 \pm 0.27(-0.12$ to 0.00$)$ & .06 \\
$J_{0}$ & $0.02 \pm 0.16(-0.02$ to 0.06$)$ & .29 & $0.05 \pm 0.25(0.00$ to 0.12$)$ & .08 & $0.03 \pm 0.23(-0.02$ to 0.09$)$ & .20 \\
$J_{45}$ & $0.00 \pm 0.22(-0.06$ to 0.05$)$ & .86 & $0.00 \pm 0.22(-0.05$ to 0.05$)$ & .96 & $0.00 \pm 0.06(0.00$ to 0.02$)$ & .42 \\
\hline
\end{tabular}

$\mathrm{ARK}$ = autorefractor; $\mathrm{Cl}=$ confidence interval; $\mathrm{M}=$ spherical equivalent; $\mathrm{RET}=$ retinoscopy; $\mathrm{SUB}=$ subjective refraction.

*Statistical significance. 


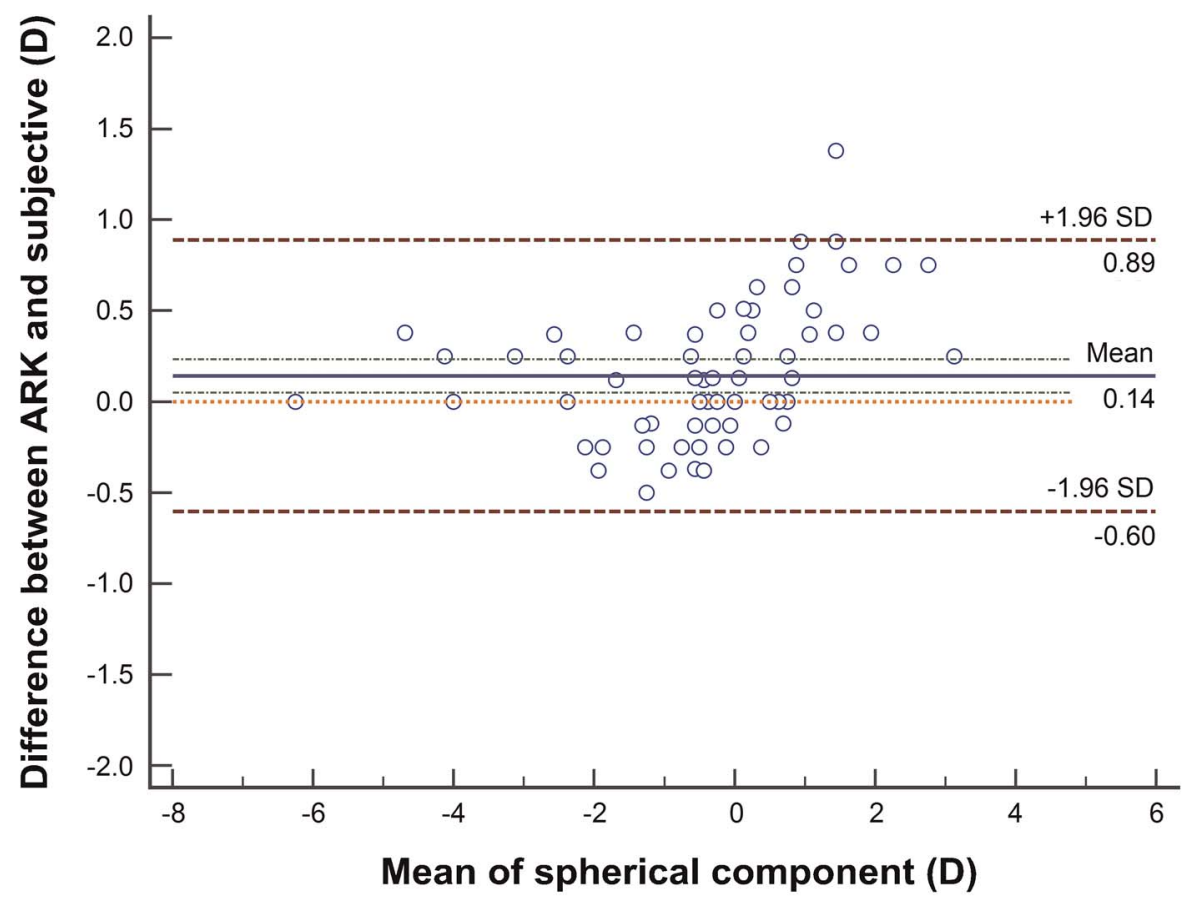

FIGURE 1. Bland-Altman plots of the spherical component between autorefraction and subjective refraction. For all the Bland-Altman plots, the solid blue lines represent the observed mean agreement between methods, dashed green lines above and below the blue line represent $95 \% \mathrm{Cl}$ of the observed mean, dashed brown lines represent 95\% limits of agreement, and dashed orange horizontal lines represent perfect mean agreement between methods. ARK = autorefractor $\mathrm{Cl}=$ confidence interval.

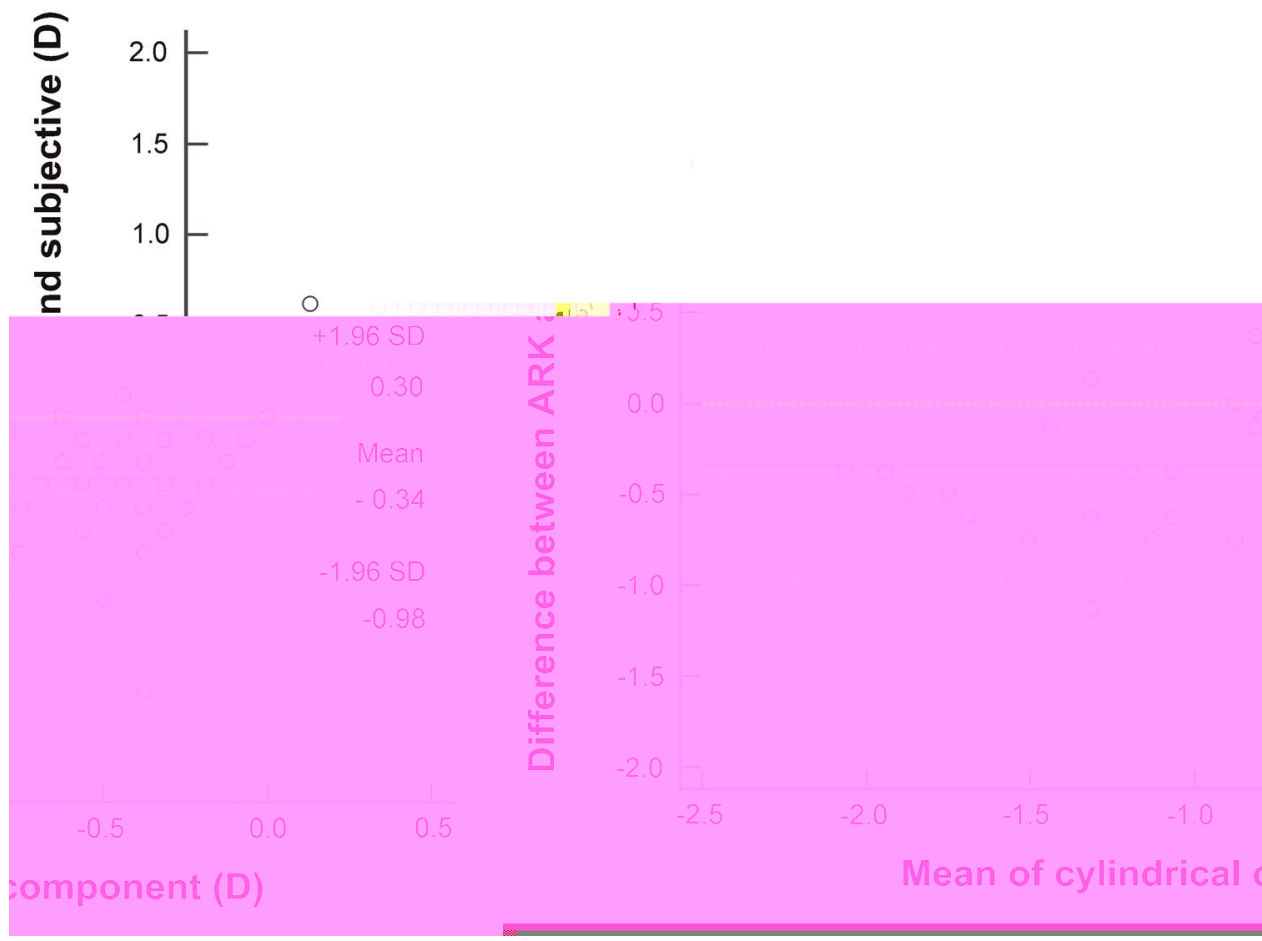

FIGURE 2. Bland-Altman plots of the spherical equivalent between autorefraction and subjective refraction. 
ARK and subjective refraction, and retinoscopy and subjective refraction were -0.60 to $0.80,-0.60$ to 0.89 , and -0.46 to 0.55 diopter, respectively (Fig. 1).

\section{Spherical Equivalent Analysis}

No significant differences were observed in spherical equivalent findings between any of the three methods (Table 1). The 95\% limits of agreement between the ARK and retinoscopy, the ARK and subjective refraction, and retinoscopy and subjective refraction were -0.65 to $+0.65,-0.80$ to +0.69 (Fig. 2 ), and -0.59 to +0.47 diopter.

\section{Cylindrical Component Analysis}

The ARK statistically significantly overestimated the cylindrical component compared with both retinoscopy and subjective refraction, with the largest difference observed between the ARK and subjective refraction (0.33 diopter cylinder; Table 1; Figs. 3, 4). The 95\% limits of agreement between the ARK and retinoscopy, the ARK and subjective refraction, and retinoscopy and subjective refraction were -0.67 to $0.37,-0.98$ to 0.30 (Fig. 3), and -0.80 to 0.43 diopter, respectively.

\section{Vector Analysis}

The mean horizontal cylindrical vector $\left(J_{0}\right)$ differences between the ARK and retinoscopy, retinoscopy and subjective refraction, and the ARK and subjective refraction were not statistically significant (Table 1). The ARK measurements were slightly more negative than retinoscopy and subjective refraction. The $95 \%$ limits of agreement between the ARK and retinoscopy, the ARK and subjective refraction, and retinoscopy and subjective refraction were -0.30 to 0.34 , -0.47 to 0.58 (Fig. 4), and -0.43 to 0.58 diopter, respectively. Although the ARK readings were slightly biased toward negative cylinder, the mean differences in the oblique cylindrical vector $\left(J_{45}\right)$ between the ARK, retinoscopy, and subjective refraction were not statistically significant (Table 1). The 95\% limits of agreement between the ARK and retinoscopy, the ARK and subjective refraction, and retinoscopy and subjective refraction were -0.44 to $+0.44,-0.44$ to +0.44 (Fig. 5), and -0.12 to +0.13 diopter.

\section{Variability}

The intratest and intertest variability of the ARK-1 was small (Table 2).

\section{DISCUSSION}

In general, the levels of agreement between autorefraction and subjective refraction measurements were in accordance with previous studies. Certainly, the spherical component and mean spherical equivalent measurement differences between the ARK and subjective refraction are similar to those observed for other models of autorefractor such as Shin-Nippon 500, Nidek AR-1000, Allergan Humphrey 570, Allergan Humprey 500, and Canon RK-1 autorefractor. $7,8,11,12,21,22$ Retinoscopy and subjective refraction exhibited the highest levels of agreement across all cutoff values herein. More importantly, however, the agreement was least between the ARK and subjective refraction measurements, which are the most relevant clinical parameters when determining the level of confidence a clinician should afford to a new autorefractor device. Our findings indicate that this autorefractor differs by more than 0.25 diopter for close to one in every two measurements and more than 0.50 diopter for one in five (Table 3). Although these findings are not surprising given the various factors that affect both types of measurements, they are clinically important and should be considered in routine clinical practice.

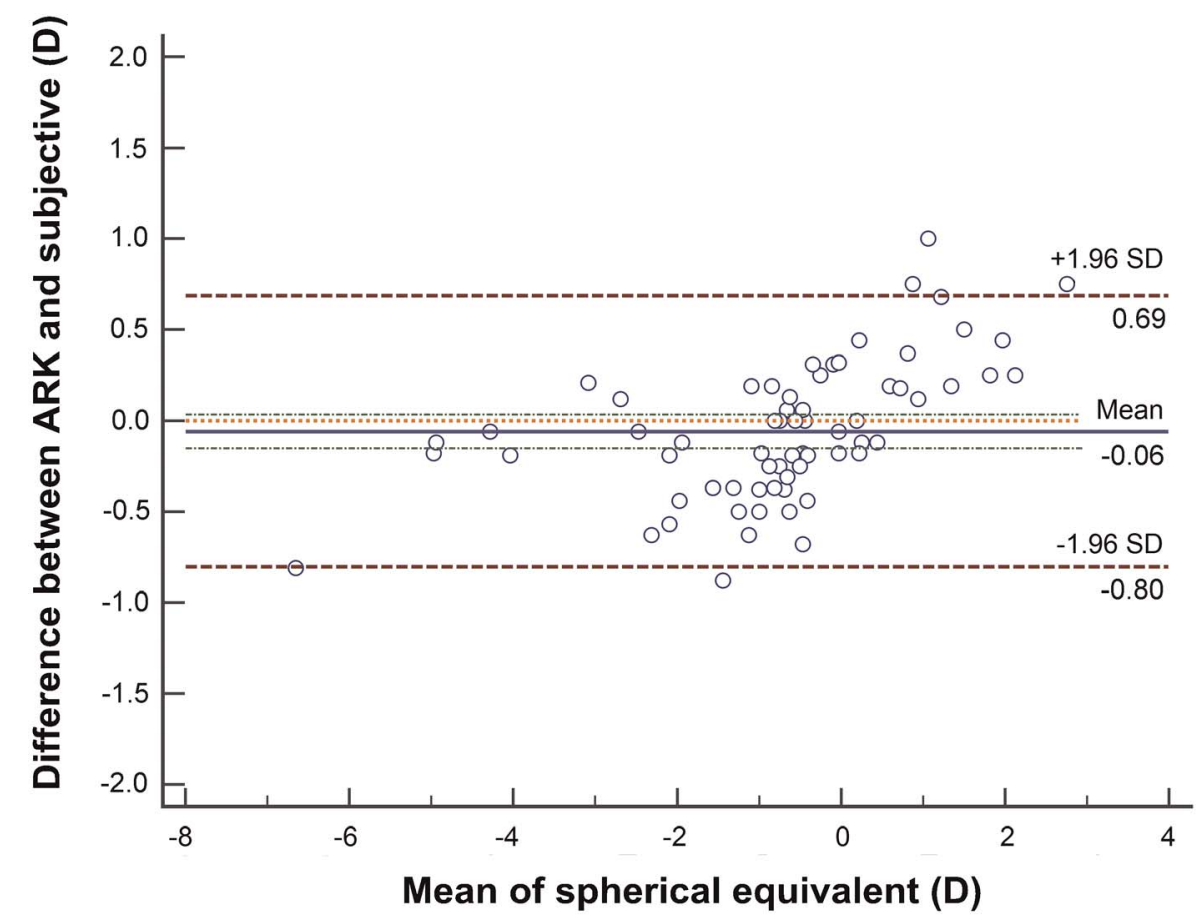

FIGURE 3. Bland-Altman plots of the cylinder between autorefraction and subjective refraction. 


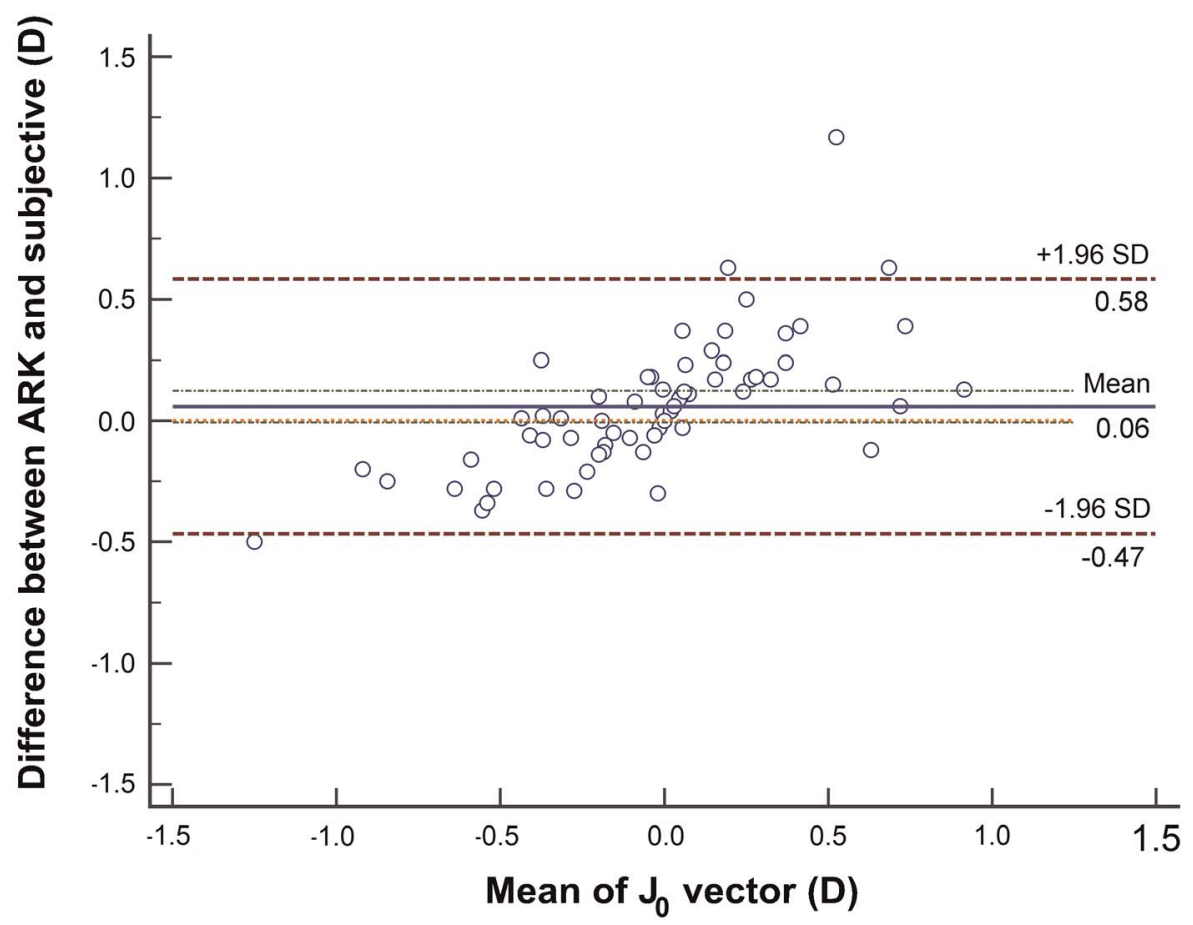

FIGURE 4. Bland-Altman plots of the $J_{0}$ vector between autorefraction and subjective refraction.

The positive bias of the spherical component refractive error with the ARK as compared with subjective refraction was statistically significant but not clinically significant (it is a common practice for clinicians to prescribe in 0.25-diopter steps). The direction of bias (positive bias) found herein is similar to that found with the ShinNippon autorefractor, an autorefractor with binocular viewing design to allow for minimal influence of accommodation on refractive measurements. ${ }^{9}$ This finding could imply that the accommodation

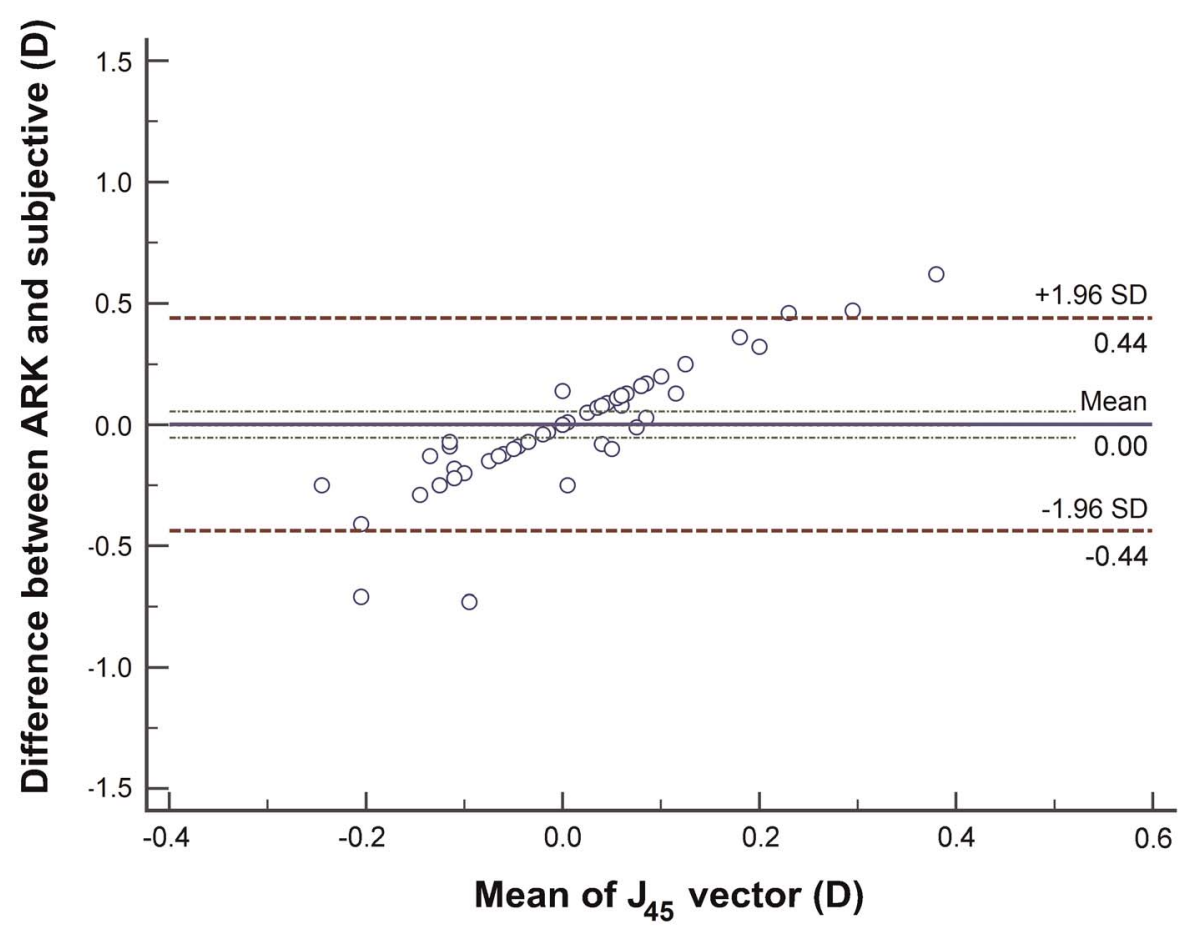

FIGURE 5. Bland-Altman plots of the $J_{45}$ vector between autorefraction and subjective refraction. 
TABLE 2. Intratest (repeatability) and intertest variability

(reproducibility) of the ARK-1 in 28 eyes of 14 individuals

\begin{tabular}{lcccc}
\hline & & \multicolumn{3}{c}{ Intertest variability } \\
\cline { 3 - 6 } Refractive components & $\begin{array}{c}\text { Intratest variability } \\
\text { DS or DC }\end{array}$ & $\begin{array}{c}\text { Mean difference } \\
\text { (DS or DC) }\end{array}$ & SD & $\boldsymbol{P}$ \\
\hline Sphere & 0.15 & 0.03 & 0.33 & .57 \\
Cylinder & 0.10 & -0.03 & 0.27 & .49 \\
Spherical equivalent & 0.13 & 0.01 & 0.30 & .75 \\
$J_{0}$ & 0.06 & 0.02 & 0.16 & .49 \\
$J_{45}$ & 0.05 & 0.007 & 0.09 & .64 \\
\hline
\end{tabular}

$\mathrm{DC}=$ diopter cylinder; $\mathrm{DS}=$ diopter sphere.

control mechanism (fogging) incorporated within the ARK-1 compares relatively favorably with binocular viewing design autorefractors. The magnitude of positive bias in the spherical component between ARK and subjective refraction observed herein is also broadly similar to that reported with other devices $(0.15$ to 0.18$),{ }^{7,9}$ being slightly higher than some (0.005 to 0.04 diopter sphere), ${ }^{23}$ but lower in terms of mean spherical equivalent for other studies ( 0.15 to 0.16 diopter sphere). ${ }^{9}$ Because cycloplegia was not used in this study, the positive slopes of the bias in the Bland-Altman plots for sphere and spherical equivalent measurement between the methods (Figs. 1,2) could be due to the fogging mechanism of the instrument or an accommodative effect in younger hyperopic participants during subjective refraction or a combination of both. However, because hyperopes comprised only $22 \%$ of study participants and were almost exclusively older than 40 years (97\%), a strong accommodative effect is unlikely.

It must be noted, however, that, although the mean differences of refractive components between the tests seem to be small, their agreement must be interpreted with caution. For instance, if we consider the spherical equivalent comparison between the ARK and subjective refraction, we observe a nonsignificant mean difference of 0.04 diopter sphere. If we look at the Bland-Altman plot (Fig. 1), however, the wide limits of agreement could be clinically important (95\% limits of agreement, -0.80 to +0.69 diopter). In fact, the limits of agreement between the ARK and subjective refraction are the widest compared with other test pairs. The nonsignificant difference between the mean spherical equivalent values is potentially due to the nature of the bias in the ARK measurements wherein the hyperopic and cylindrical values are overestimated, which then cancel each other out. Similar finding is also observed in the spherical component measures between the ARK and subjective refraction (Table 1, Fig. 3). The limits of agreement between the ARK and subjective refraction in the spherical and spherical equivalent measurements, however, were similar to some of the previous designs of autorefractors ${ }^{11}$ but considerably higher than others. ${ }^{7,8}$
With regard to the cylindrical component, the positive bias between autorefractor and subjective refraction (-0.33 diopter cylinder) was considerably higher than in previous studies ( 0.002 to $0.05 \mathrm{di}$ opter cylinder). ${ }^{8,21,22}$ The bias between retinoscopy and subjective refraction was also high compared with earlier studies. ${ }^{1,10}$ Both the autorefractor and retinoscopy measurements for the $J_{0}$ and $J_{45}$ components, meanwhile, were less positively biased compared with subjective refraction measurements relative to those obtained in previous studies with different models of autorefractor,, 22 whereas the percentage of cylindrical component differences between ARK and subjective refraction that fell within \pm 0.25 and \pm 0.50 diopter cylinder was considerably lower $(74$ to $87 \%$ for \pm 0.25 diopter cylinder and 90 to $97 \%$ for \pm 0.50 diopter cylinder in previous studies ${ }^{21,22}$; Table 3 ).

The levels of intertest and intratest variability of the ARK-1 are in agreement with previous validation studies on Shin-Nippon SRW-5000, ${ }^{8}$ the Hoya AR-570, ${ }^{10}$ and the Nikon NRK8000. ${ }^{24}$

The reasons underlying the observed variation in findings between different methods could be many. Previous researchers have suggested that the presence of higher-order aberrations can lead to significant differences in measurements between different methods. ${ }^{25}$ In addition, the three refraction methods pose different accommodative demands to the patients that influence the refraction measurement, hence possibly explaining some inconsistencies in the results. Moreover, the cognitive demand involved in subjective refraction, unlike in either of the other two refraction methods, can at least in part contribute to the differences. Likewise, the discrepancy in the measurements in our study compared with other studies could relate to the different principles used in different models of autorefractors. For instance, the ARK-1 uses the two-pinhole Schiener disc principle, whereas other autorefractors such as Canon use the ray deflection method, Topcon uses the image size principle, Humphrey autorefractor uses the knife edge principle, Shin-Nippon (Grand Seiko) uses the image reflection method, and the Nidek OPD Scan II uses the retinoscopy principle. ${ }^{14,26}$ Moreover, different models of autorefractors have different mechanisms to control accommodation such as positioning of the fixation target (internal vs. external) and viewing condition (monocular vs. binocular).

All of the refractive components obtained via retinoscopy were closer to subjective refraction than with the autorefraction results. In addition, the range of limits of agreement between the retinoscopy and subjective refraction was narrower than that between the ARK and subjective refraction. This suggests that retinoscopy when performed by an experienced clinician can be the method of choice for the starting point of subjective refraction. This finding is in accordance with an earlier study. ${ }^{1}$ However, the small intratest and intertest variability of the ARK-1 may signify that this instrument can be used in various other purposes such as for studies observing the change in refractive errors in myopia control studies,

TABLE 3. Proportion of the difference in refractive components between the three methods

\begin{tabular}{|c|c|c|c|c|c|c|c|c|c|}
\hline \multirow[b]{2}{*}{ Refractive components } & \multicolumn{3}{|c|}{ ARK and SUB (\%) } & \multicolumn{3}{|c|}{ ARK and RET (\%) } & \multicolumn{3}{|c|}{ RET and SUB (\%) } \\
\hline & \pm 0.25 & \pm 0.50 & \pm 1.00 & \pm 0.25 & \pm 0.50 & \pm 1.00 & \pm 0.25 & \pm 0.50 & \pm 1.00 \\
\hline Sphere & 60 & 86 & 98 & 64 & 92 & 98 & 80 & 97 & 100 \\
\hline Spherical equivalent & 56 & 85 & 98 & 63 & 91 & 98 & 74 & 94 & 100 \\
\hline Cylinder & 46 & 77 & 97 & 73 & 92 & 100 & 72 & 86 & 100 \\
\hline
\end{tabular}


screening children for refractive errors, and ensuring findings obtained with retinoscopy in highly irregular/aberrated eyes.

It must be acknowledged that this study is not without limitations. First, we agree with previous authors that considering subjective refraction as a reference may have limitations, ${ }^{22,27}$ as there may be a variation in measurements if subjective refraction is conducted by one or more clinicians. ${ }^{10}$ Therefore, we considered subjective refraction by a single practitioner to minimize clinician bias, as also implemented by other studies. ${ }^{22,28}$ Second, given the potential of ARK-1 to measure spherical refractive error within the range of -30 to +25 diopters and cylindrical error of up to 12 diopters, the narrow range of refractive errors included in our study limits any interpretation of the utility of ARK-1 in patients with high refractive errors. Previous studies have demonstrated higher variability of autorefractor measurement with high refractive error measurements, ${ }^{7}$ although it should be noted that epidemiological studies indicate that between 85 and $90 \%$ of population refractive errors lie within the measurement range reported herein, so the results are applicable for most of likely clinic attendees. ${ }^{29,30}$ Third, the researcher had knowledge of the participant's previous prescription, which may have introduced some bias in the subjective refraction. The researcher made sure, however, to follow a consistent routine for each participant, with the end point defined by the criteria to achieve the best possible acuity and comfortable vision with the new prescription. A standardized method of subjective refraction including alternate occlusion for binocular balancing was therefore conducted on all participants irrespective of the knowledge of the participants' previous prescription. Finally, this study involved the use of a single ARK-1 instrument, so the findings cannot be assumed to reflect performance limits of all ARK-1 instruments.

In conclusion, Nidek ARK-1 could be a valuable addition to any optometric or ophthalmic practice as an instrument for approximating refractive errors and tracking the progression of refractive development in adults. Some caution should be exercised while interpreting its results, which, as with all autorefractors, should not be used as a replacement for traditional prescribing routines.

\section{ARTICLE INFORMATION}

Submitted: July 11, 2018

Accepted: February 28, 2019

Funding/Support: None of the authors have reported funding/support.

Conflict of Interest Disclosure: None of the authors have reported a financial conflict of interest.

Author Contributions and Acknowledgments: Conceptualization: NP; Data Curation: NP, SA; Formal Analysis: NP Investigation: NP, SA, AT; Methodology: NP; Supervision: BS, JL; Visualization: NP; Writing - Original Draft: NP Writing - Review \& Editing: SA, AT, BS, JL.

We would like to thank Dr. Mark Bullimore, MCOptom, $\mathrm{PhD}, \mathrm{FAAO}$, for his contributions to the early development of the article.

\section{REFERENCES}

1. Jorge J, Queirós A, Almeida JB, et al. Retinoscopy/ Autorefraction: Which is the Best Starting Point for a Noncycloplegic Refraction. Optom Vis Sci 2005;82:64-8.

2. Strang NC, Gray LS, Winn B, et al. Clinical Evaluation of Patient Tolerance to Autorefractor Prescriptions. Clin Exp Optom 1998;81:112-8.

3. Wolffsohn JS, Gilmartin B, Mallen EA, et al. Continu ous Recording of Accommodation and Pupil Size Using the Shin-Nippon SRW-5000 Autorefractor. Ophthalmic Physiol Opt 2001;21:108-13.

4. Zadnik K. The Glenn A. Fry Award Lecture (1995) Myopia Development in Childhood. Optom Vis Sci 1997;74:603-8.

5. Hung GK, Ciuffreda KJ, Rosenfield M. Proximal Contribution to a Linear Static Model of Accommodation and Vergence. Ophthalmic Physiol Opt 1996;16:31-41.

6. Rosenfield M, Ciuffreda KJ. Effect of Surround Propinquity on the Open-loop Accommodative Response. Invest Ophthalmol Vis Sci 1991;32:142-7.

7. Mallen EH, Wolffsohn JS, Gilmartin B, et al. Clinical Evaluation of the Shin-Nippon SRW-5000 Autorefractor in Adults. Ophthalmic Physiol Opt 2001;21:101-7.
8. Mallen EH, Gilmartin B, Wolffsohn JS, et al. Clinical Evaluation of the Shin-Nippon SRW-5000 Autorefractor in Adults: An Update. Ophthalmic Physiol Opt 2015;35:622-7.

9. Davies LN, Mallen EH, Wolffsohn JS, et al. Clinical Evaluation of the Shin-Nippon NVision-K 5001/Grand Seiko WR-5100K Autorefractor. Optom Vis Sci 2003; 80:320-4.

10. Bullimore MA, Fusaro RE, Adams CW. The Repeatability of Automated and Clinician Refraction. Optom Vis Sci 1998;75:617-22.

11. Kinge B, Midelfart A, Jacobsen G. Clinical Evaluation of the Allergan Humphrey 500 Autorefractor and the Nidek AR-1000 Autorefractor. Br J Ophthalmol 1996;80:35-9.

12. Raj PS, Villada JR, Lewis $A E$, et al. Comparative Evaluation of the Allergan Humphrey 570 and Canon RK-1 Autorefractors: I. Objective Autorefraction in Normal Subjects. Eye 1992;6:284-6.

13. McCaghrey GE, Matthews FE. Clinical Evaluation of a Range of Autorefractors. Ophthalmic Physiol Opt 1993;13:129-37.

14. Kaschke M, Donnerhacke KH, Rill MS. Optical Devices in Ophthalmology and Optometry: Technology, Design Principles and Clinical Applications. Weinheim: Wiley-VCH; 2014.

15. NIDEK Co., Ltd. Auto Ref/Keratometer ARK-1s/1a/1l Auto Refractometer/Auto Keratometer/Non-contact Tonometer/Pachymeter. Available at: http://www. nidekintl.com/product/ophthaloptom/refraction/ref_ auto/ark-1s.html. Accessed June 10, 2018.

16. Faul F, Erdfelder E, Lang A-G, et al. G*Power 3: A Flexible Statistical Power Analysis Program for the Social, Behavioral, and Biomedical Sciences. Behavior Research Methods, 2007;39:175-91.

17. Armstrong RA. Statistical Guidelines for the Analysis of Data Obtained from One or Both Eyes. Ophthalmic Physiol Opt 2013;33:7-14.

18. Thibos LL, Wheeler W, Horner D. Power Vectors: An Application of Fourier Analysis to the Description and Statistical Analysis of Refractive Error. Optom Vis Sci 1997;74:367-75.
19. Bland JM, Altman DG. Measuring Agreement in Method Comparison Studies. Stat Methods Med Res 1999;8:135-60.

20. Schoonjans F, Zalata A, Depuydt CE, et al. MedCalc: A New Computer Program for Medical Statistics. Comput Methods Programs Biomed 1995; 48:257-62.

21. Shneor E, Millodot M, Avraham O, et al. Clinical Evaluation of the L80 Autorefractometer. Clin Exp Optom 2012;95:66-71.

22. McGinnigle S, Naroo SA, Eperjesi F. Evaluation of the Auto-refraction Function of the Nidek OPD-Scan III. Clin Exp Optom 2014;97:160-3.

23. Sheppard AL, Davies LN. Clinical Evaluation of the Grand Seiko Auto Ref/Keratometer WAM-5500. Ophthalmic Physiol Opt 2010;30:143-51.

24. Elliott M, Simpson T, Richter D, et al. Repeatability and Accuracy of Automated Refraction: A Comparison of the Nikon NRK-8000, the Nidek AR-1000, and Subjective Refraction. Optom Vis Sci 1997;74:434-8.

25. Jinabhai A, O'Donnell C, Radhakrishnan H. A Comparison between Subjective Refraction and Aberrometryderived Refraction in Keratoconus Patients and Control Subjects. Curr Eye Res 2010;35:703-14.

26. Keirl A, Christie C. Clinical Optics and Refraction: A Guide for Optometrists, Contact Lens Opticians and Dispensing Opticians. Philadelphia: Elsevier Health Sciences; 2007

27. McBrien NA, Millodot M. Clinical Evaluation of the Canon Autoref R-1. Optom Vis Sci 1985;62:786-92.

28. Pesudovs K, Weisinger HS. A Comparison of Autorefractor Performance. Optom Vis Sci 2004;81:554-8.

29. Kempen JH, Mitchell P, Lee KE, et al. The Prevalence of Refractive Errors among Adults in the United States, Western Europe, and Australia. Arch Ophthalmol 2004;122:495-505.

30. Wolfram C, Höhn R, Kottler U, et al. Prevalence of Refractive Errors in the European Adult Population: The Gutenberg Health Study (GHS). Br J Ophthalmol 2014;98:857-61. 